\title{
Association Game for Conflict Resolution between UEs and Small Cells
}

\author{
Jaesung Park \\ School of Information Convergence, Kwangwoon University, 20 Kwangwoon-ro, Nowon-gu, Seoul 01897, Republic of Korea \\ Correspondence should be addressed to Jaesung Park; jaesungpark@kw.ac.kr
}

Received 19 February 2020; Revised 15 May 2020; Accepted 25 May 2020; Published 9 June 2020

Academic Editor: Hideyuki Takahashi

Copyright (C) 2020 Jaesung Park. This is an open access article distributed under the Creative Commons Attribution License, which permits unrestricted use, distribution, and reproduction in any medium, provided the original work is properly cited.

\begin{abstract}
Small cells are expected to increase the capacity and the coverage of a wireless network. Since the transmission ranges of the small cells overlap with each other, a user equipment (UE) can be located within the service areas of multiple cells. Therefore, the association decisions made by the small cells and the UEs influence the energy efficiency of the cells and the data rates provided to the UEs. However, if they attempt to achieve their goals selfishly, the network may become instable in that a UE has to change its associated cell and a cell has to change the set of UEs associated with it frequently. To tackle the problem, in this paper, we propose an association game between UEs and cells. We cast the problem as a matching game where each player has preference over the other player. Then, we propose an association algorithm composed of a UE-side part and a cell-side part. Through simulation studies, we show that the proposed method outperforms the other methods in that it can enhance the average energy efficiency of the small cell network and the data rates that UEs can obtain from the network.
\end{abstract}

\section{Introduction}

Data traffic exchanged through cellular networks has increased drastically over the recent years. According to Ericsson mobility report [1], mobile data traffic grows 82 percent between the first quarter in 2018 and the first quarter in 2019 , and it is expected that the mobile data will grow 30 percent annually until 2024. In addition, the proportion taken by video traffic is also expected to increase. Video traffic took 60 percent of the $28 \mathrm{~EB}$ mobile data traffic per month in 2018 , and it is forecasted that video traffic will take 74 percent of the $131 \mathrm{~EB}$ data traffic per month by 2024 .

To accommodate the traffic growth and data hungry applications, cellular networks have been evolved by increasing the capacity and the coverage. For example, ITU-R specified the enhanced mobile broadband (eMBB) as one of the important $5 \mathrm{G}$ and beyond cellular network service categories. To increase the capacity of wireless network, various methods have been devised (for example, new radio [2], advanced interference management [3], and network slicing [4]). Among those, small cells are expected to increase both the capacity and the coverage of the network in a cost and energy-effective manner $[5,6]$. By deploying small cells densely, the downlink data rate provided to each UE (User Equipment) will be enhanced. However, as the number of small cells increases, the energy consumed by the network increases accordingly. Thus, energy efficient operation becomes one of the important design requirements of a small cell network [7].

Cell sleep methods have been proposed to increase the energy efficiency (EE) of a small cell network $[8,9]$. The basic idea of the cell sleep is to switch off small cells when they are not necessary to provide reasonable services to the UEs having data to send. After determining cells to sleep, the cell sleep method forces the UEs connected to the sleeping cell to associate with new active cells. Power control has also been used widely to increase the EE of a network $[10,11]$. The signal quality increases as the transmission power increases. However, the amount of interferences also increases with the transmission power, which results in decreasing the throughput of a network. Thus, the dynamic power control method seeks to find the optimal transmission power for 
each cell in the network so that the energy efficiency of the network is maximized.

On the other hand, the purpose of UEs is to increase the service rates received from the network. In a small cell network, a UE is within the transmission ranges of multiple cells. Thus, the cell association method will significantly influence the data rates received by a UE. Conventionally, a UE monitors the signal strengths from neighboring cells and attempts to associate with the cell giving the highest signal strength. However, since a UE is not aware of the load condition of a cell, the cell giving the highest signal strength to a UE is not necessarily the one that can provide the highest data rate to the UE. The authors in [12] proposed an association method to balance the loads of the cells in the network. An interference model is proposed in [13] to predict the cell load. Using the estimated load, the authors proposed a joint optimization algorithm for user association and antenna tilt adjustment.

Through association management, a cell tries to enhance its EE while UEs attempt to maximize the data rate received from a network. Since the intention of the two entities (i.e., the cells and the UEs) may be conflicting, each entity may not be able to accomplish their goals if a cell and a UE try to achieve their goals selfishly. Therefore, the small cell network can be instable in that UEs and cells keep changing their associations.

To resolve the issue, we propose a matching game between UEs and cells. UEs and cells have the most wanted counterpart to achieve their goals. Therefore, we cast the association problem as a matching problem between UEs and cells who have preferences over the others. Then, we devise an association algorithm that results in stable pairs of UEs and cells by matching each UE to its most preferred achievable cell. Through simulation studies, we show that the proposed method can increase both the EEs of the cells and the downlink data rate provided to UEs, compared with those acquired by the conventional methods.

The rest of the paper is organized as follows: after, we formulate the problem by describing the system model in Section 2; we present the proposed algorithm in Section 3. We evaluate the proposed method through simulation studies in Section 4 and conclude the paper in Section 5.

\section{System Model}

We consider a wireless network where small cells are deployed densely. Before we proceed, we present the notations used in this paper in Table 1.

We denote by $C$ a set of small cells in the network. We assume that small cells are using the OFDMA and the timefrequency resources that a cell $i \in C$ can allocate to UEs are divided into the physical resource blocks (PRBs). We denote by $B_{P}$ the bandwidth of a PRB and by $B_{M}$ the maximum number of PRBs that a cell has. We assume that the frequency reuse factor in the network is one. We denote the set of UEs deployed in the network as $U$. We also denote by $U_{i}$ the set of UEs associated with a cell $i$ and by $C_{u}$ the set of cells that a UE $u$ can make an association with.
TABLe 1: Notations used.

\begin{tabular}{lr}
\hline Notations & Meaning \\
\hline$U_{i}$ & Set of UEs associated with a cell $i$. \\
$C_{u}$ & Set of cells that a UE $u$ can make an association with. \\
$B_{P}$ & Bandwidth of a physical resource block. \\
$B_{M}$ & The maximum number of a physical resource block in \\
$\beta_{u, i}$ & a cell. \\
$\gamma_{u, i}$ & DINR between a UE $u$ and a cell $i$. \\
$\Gamma_{i}$ & Total data rate that a cell $i$ can provide. \\
$E_{i}$ & Total energy consumed by a cell $i$. \\
$\rho_{i}$ & Load of a cell $i$. \\
$\Psi_{i}$ & Energy efficiency of a cell $i$. \\
$\Omega$ & Total energy efficiency of a system. \\
\hline
\end{tabular}

Let us consider the situation where a UE $u$ is associated with a cell $i$. If the transmission power of a cell $i$ is $p_{i}$, the power received at $u$ becomes

$$
\alpha_{u, i}=p_{i} h_{u, i}
$$

where $h_{u, i}$ is the path gain between $u$ and $i$. The path gain includes all the factors that can influence the received signal power such as path loss, shadowing, and antenna height. Then, the SINR (signal-to-interference-plus-noise-ratio) between $u$ and $i$ is expressed as

$$
\beta_{u, i}=\frac{\alpha_{u, i}}{\sum_{j \in C-\{i\}} \alpha_{u, j}+N_{0}},
$$

where $N_{0}$ is a noise power.

The data rate that a cell $i$ can provide to a UE $u$ depends on the amount of resources that $i$ allocates to $u$ and the SINR between them. We denote by $m_{u, i}$ the number of PRBs that $i$ allocates to $u$. Then, by the Shannon's capacity formula, the downlink data rate provided to $u$ by $i$ is given as

$$
\gamma_{u, i}=m_{u, i} B_{P} \log _{2}\left(1+\beta_{u, i}\right)
$$

$m_{u, i}$ depends on the scheduler used by a cell $i$ and the number of UEs being served by $i$. Since we focus on the association management method in this paper, we assume that each cell serves the UEs associated with it in a round-robin manner. Then,

$$
m_{u, i}=\frac{B_{M}}{\left|U_{i}\right|}
$$

where $\left|U_{i}\right|$ is the cardinality of the set $U_{i}$. However, we note that the type of the scheduler does not influence the operation of the proposed method. 
From Equation (3), the total data rate that a cell can provide is obtained as

$$
\Gamma_{i}=\sum_{u=1}^{\left|U_{i}\right|} \gamma_{u, i}=\sum_{u=1}^{|U|} I_{u, i} \gamma_{u, i},
$$

where $I_{u, i}$ is the indicator function such that

$$
I_{u, i}= \begin{cases}1, & u \in U_{i} \\ 0, & \text { otherwise }\end{cases}
$$

The energy consumption model of a cell is presented in [14]. The model is composed of two parts. The first part represents the amount of energy when a cell is not involved in transmitting or receiving packets while the second part represents the amount of power consumed by a cell which depends on the load of the cell. Specifically, the total energy consumed by a cell $i$ is given as

$$
E_{i}=n_{A} P_{0}+\Delta \rho_{i} p_{i}
$$

where $P_{0}$ is the fixed amount of energy consumed by a cell, $n_{A}$ is the number of antennas of a cell, $\Delta$ is the slope of the energy consumption, and $\rho_{i}$ is the load of a cell.

The load of a cell is defined as the ratio of the amount of allocated PRBs to $B_{M}$. However, since a cell in the system allocates all the resources to UEs associated with it (Equation (4)), the load of a cell becomes 1 regardless of the number of UEs being served by the cell. To cope with the issue, we assume that there is the minimum downlink data rate $\gamma_{\text {th }}$ that makes a UE keep its current association. To define $\rho_{i}$, we let $\tilde{m}_{u, i}$ be the minimum number of PRBs needed to satisfy $\gamma_{u, i}$ $\geq \gamma_{\text {th }}$. From Equation (3), $\tilde{m}_{u, i}$ is given as

$$
\tilde{m}_{u, i}=\left\lfloor\frac{\gamma_{\mathrm{th}}}{B_{P} \log _{2}\left(1+\beta_{u, i}\right)}\right\rfloor,
$$

where $\lfloor x\rfloor$ is the smallest integer that is not smaller than $x$. Then, the load of a cell is given as

$$
\rho_{i}=\sum_{u=1}^{|U|} \frac{I_{u, i} \tilde{m}_{u, i}}{B_{M}}
$$

The energy efficiency of a cell is defined as the total data rate the cell provides over the amount of energy consumed to support the total data rate. From Equations (5) and (7), the energy efficiency of a cell is obtained as

$$
\Psi_{i}=\frac{\Gamma_{i}}{E_{i}}
$$

Using Equation (10), we get the total energy efficiency of a system.

$$
\Omega=\sum_{i=1}^{|C|} \sum_{u=1}^{|U|} \frac{\mathrm{I}_{u, i} \gamma_{u, i}}{n_{A} P_{0}+\Delta \rho_{i} p_{i}} .
$$

We note that the set of associations between cells and UEs (i.e., $I_{u, i}$ ) influences $\Psi_{i}$ and $\gamma_{u, i}$. In the respect of a cell, its purpose is to increase its energy efficiency. Thus, when a cell $i$ receives the association requests from UEs, it attempts to accept the UEs who can increase its energy efficiency. On the contrary, the goal of a UE is to get the downlink data rate as high as possible. From Equation (3), we see that $\gamma_{u, i}$ depends on $\beta_{u, i}$ and $m_{u, i}$. As we can see in Equation (2), the association made between a UE and a cell affects $\beta_{u, i}$. The number of PBRs allocated to a UE by a cell is also influenced by the association made between the cell and UEs (see Equation (4)). Therefore, the set of optimal associations for a cell may not be the optimal association for a UE because their purposes are different. If each entity (a cell and a UE) attempts to achieve their goals selfishly, the associations made between cells and UEs in a network may not be stabilized because UEs and cells may keep changing their associations to accomplish their goals.

To resolve the conflict, we propose an association method using a matching game theory [15]. Specifically, by noting that each party has a preferred counterpart to accomplish its purpose, we consider the association problem as the matching with preference game with two sets of players $C$ and $U$. Then, we devise a distributed algorithm that determines the association pairs between a set of small cells and a set of UEs.

\section{Association Game}

3.1. Matching Algorithm. In this section, we present our matching-based association management algorithm. The algorithm is composed of two parts. One part is performed in a UE, and the other part is operated in a cell. When a UE $u$ needs to make an association with a cell, it measures $\beta_{u, i}$ from all the cells in $C_{u}$ by monitoring the reference signals. After collecting $\beta_{u, i}, u$ sorts the cells in $C_{u}$ in a descending order according to $\beta_{u, i}$. We denote the sorted set of cells as $\tilde{C}_{u}$. Then, $u$ attempts to associate with the first cell (say, $i$ ) in $\tilde{C}_{u}$ by sending an Association Req. message to it. If the association request is accepted by $i, u$ makes an association with $i$. If the request is rejected, $u$ removes $i$ from $\tilde{C}_{u}$ and repeats the same process again. When $u$ does not make an association with a cell until $\widetilde{C}_{u}$ becomes empty, it restarts the process by measuring $\beta_{u, i}$ again. When a UE $u$ is currently associated with a cell $i$, the cell can force $u$ to handover by sending a Cell Redirect message to $u$. In that case, $u$ starts the association procedure stated above with $\tilde{C}_{u}-\{i\}$. We summarize the UE side operation in Algorithm 1.

The cell side algorithm operates as follows: when a cell $i$ receives an Association Req. message from $u$ at time $t, i$ checks whether the data rate provided to each UE already associated with it before $t$ (denoted by $U_{i}(t-1)$ ) can still be larger than $\gamma_{\text {th }}$ if it accepts the association request from $u$. Specifically, given $\tilde{U}_{i}(t)=U_{i}(t-1) \cup\{i\}, i$ calculates the 


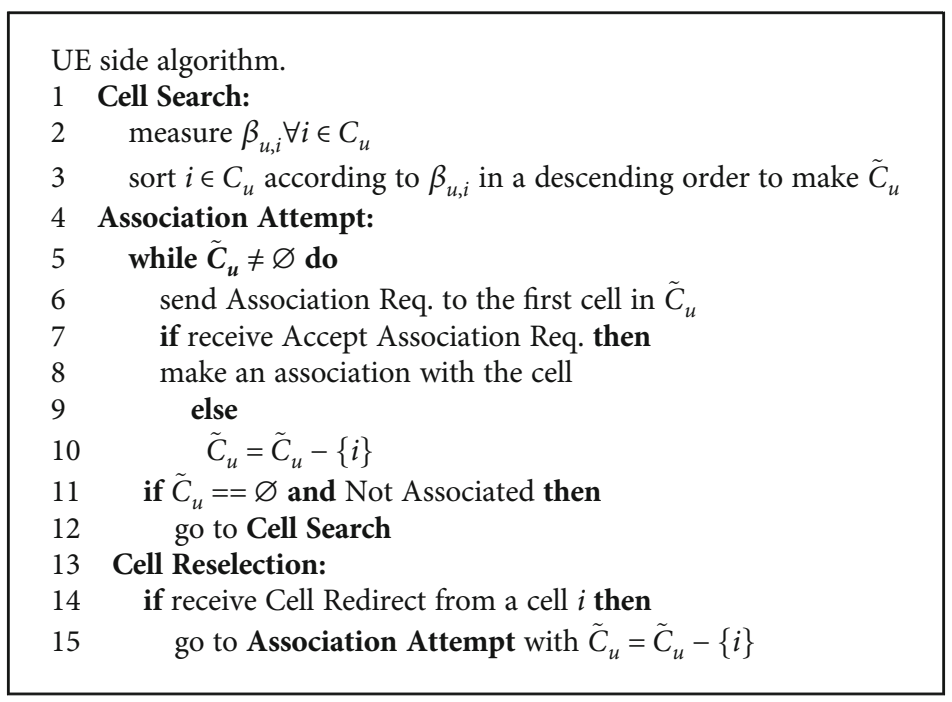

Algorithm 1:

number of PRBs allocated to a UE $v$ in $\tilde{U}_{i}(t)$ using Equation (4) as $\widehat{m}_{v, i}(t)=B_{M} /\left|\tilde{U}_{i}(t)\right|$. Then, the cell $i$ obtains the downlink data rate provided to each UE $v$ in $\tilde{U}_{i}(t)$ as $\widehat{\gamma}_{u, i}(t)=$ $\widehat{m}_{v, i}(t) B_{P} \log _{2}\left(1+\beta_{v, i}\right)$. After obtaining $\widehat{\gamma}_{u, i}(t)$, $i$ compares $\widehat{\gamma}_{u, i}(t)$ with $\gamma_{\text {th }}$. If $\widehat{\gamma}_{u, i}(t)>\gamma_{\text {th }}$, for all $v \in \tilde{U}_{i}(t)$, it means that the cell does not drive any UE in $\tilde{U}_{i}(t)$ to change its serving cell even though $i$ accepts $u$. Then, $i$ checks whether its energy efficiency can increase by accommodating $u$.

If a cell $i$ accepts the association request, the load of the cell becomes $\tilde{\rho}_{i}(t)=\sum_{v \in \tilde{U}_{i}(t)} \tilde{m}_{v, i} / B_{M}$. If we denote by $\tilde{\Psi}_{i}(t)$ the energy efficiency of a cell $i$ when it accepts the association request from $u$, using Equation (10), $i$ gets

$$
\tilde{\Psi}_{i}(t)=\frac{\sum_{v \in \tilde{U}_{i}(t)} \widehat{\gamma}_{v, i}}{n_{A} P_{0}+\Delta \tilde{\rho}_{i}(t) p_{i}} .
$$

If $\tilde{\Psi}_{i}(t)>\Psi_{i}(t-1), i$ accepts the association request from $u$ to increase its energy efficiency. On the other hand, if $\tilde{\Psi}_{i}(t) \leq \Psi_{i}(t-1)$, the energy efficiency of $i$ reduces if $i$ accepts $u$. However, instead of rejecting the association request from $u$ immediately, $i$ checks whether its energy efficiency can be increased by releasing an association with a UE in $U_{i}(t-1)$ and accepting $u$. Specifically, let us denote $U_{i, v}(t)=U_{i}(t-1) \cup\{u\}-\{v\}\left(v \in U_{i}(t-1)\right)$. Then, for all $v \in U_{i}(t-1), i$ obtains its energy efficiency when the set of UEs associated with it is $U_{i, v}(t)$ as follows.

$$
\Psi_{i, v}(t)=\frac{\sum_{k \in U_{i, v}(t)} \gamma_{k, i}}{n_{A} P_{0}+\Delta \rho_{i, v} p_{i}},
$$

where $\rho_{i, v}$ is the load of a cell when the set of UEs associated with $i$ is $U_{i, v}(t)$. A cell $i$ searches for a UE $v^{*}$ that can maximize the $\Psi_{i, v}(t)$. In other words,

$$
v^{*}=\arg \max _{v \in U_{i}(t-1)} \Psi_{i, v}(t) .
$$

Thus, the maximum energy efficiency that the cell $i$ can achieve by replacing a UE in $U_{i}(t-1)$ with $u$ becomes $\Psi_{i, v^{*}}$ $(t)$. Then, the condition $\Psi_{i, v^{*}}(t)>\Psi_{i}(t-1)$ means that the energy efficiency of a cell $i$ at time $t-1$ (i.e., $\Psi_{i}(t-1)$ ) is smaller than the energy efficiency that $i$ can get by releasing an association with $v^{*}$ and accepting the association request from $u$. Thus, a cell $i$ accepts the association request from $u$ and releases an association with $v^{*}$ by sending a Cell Redirect message to $v^{*}$. On the contrary, if $\Psi_{i, y^{*}}(t) \leq \Psi_{i}(t-1)$, $i$ cannot get a gain in terms of its energy efficiency. Therefore, $i$ rejects the association request by sending a Reject Association Req. to $u$. We show the algorithm operating in a cell in Algorithm 2.

3.2. Stability Analysis. To prove the stability of the proposed algorithm, we introduce the following definition.

Definition 1. A member $x$ in one set said to be acceptable to a member $y$ in the other set if $y$ prefers $x$ at least as well as to be not associated at all.

We state the stability of the proposed algorithm with the following proposition.

Proposition 2. The set of associations between the UEs in $U$ and the cells in $C$ is a stable matching $\mu_{s}$ in that all the UEs and cells are associated with their mutually acceptable counterparts.

Proof. Let us consider the case that the matching $\mu_{s}$ achieved by the proposed method does not associate a UE $u$ with a cell $i$, and $i$ prefers $u$ to $v \in U_{i}$. It means either $i$ is not acceptable to $u$ or $i$ rejects the association request from $u$. If $i$ is not acceptable to $u, u$ did not attempt to make an association with $i$. If $i$ rejects the association request from $u$, it negates the statement that $i$ prefers $u$ to $v \in U_{i}$. Thus, $\mu_{s}$ does not associate $i$ with a UE to whom $i$ is not acceptable. In addition, $\mu_{s}$ associates $i$ with $v$ acceptable to $i$. 


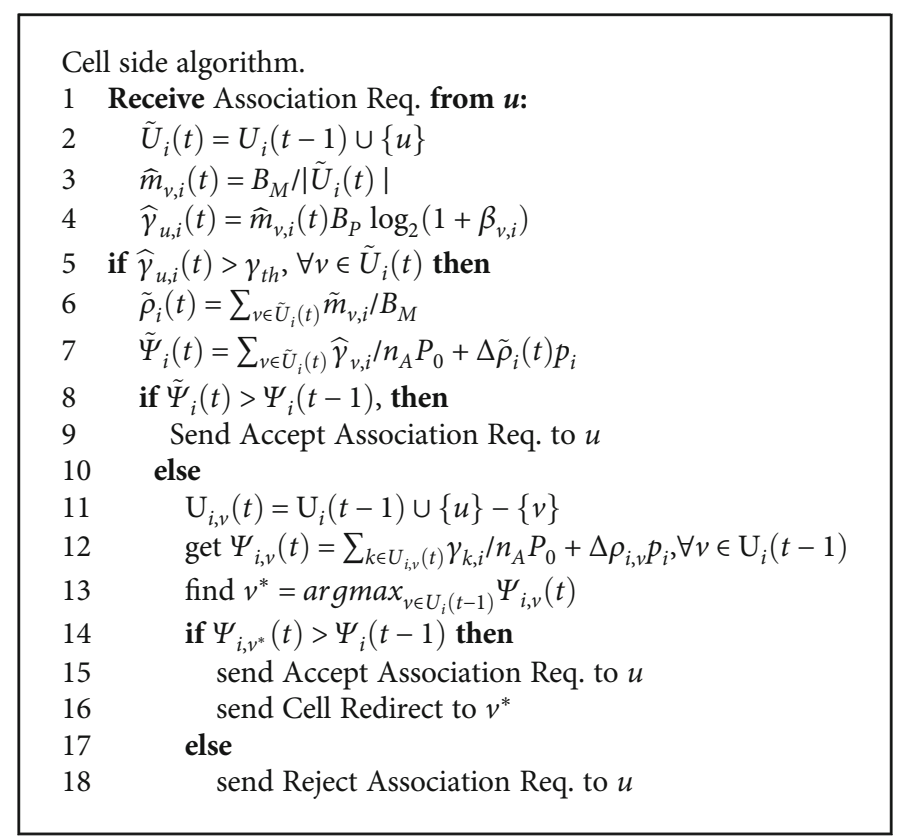

Algorithm 2:

TABLE 2: Simulation parameters.

\begin{tabular}{lc}
\hline Parameters & Values \\
\hline Simulation area $(Y)$ & $100 \times 100 \mathrm{~m}^{2}$ \\
Density of small cells & $200 / Y$ \\
System bandwidth & $5 \mathrm{MHz}$ \\
Bandwidth of a PRB & $180 \mathrm{KHz}$ \\
Max. number of PRBs in a cell & 25 \\
Tx power of a cell & $21 \mathrm{dBm}$ \\
Antenna gain of a cell & $3.0 \mathrm{dBi}$ \\
Antenna gain of a UE & $2.0 \mathrm{dBi}$ \\
UE scheduling policy & Round-Robin \\
$n_{A}$ & 1 \\
$P_{0}$ & $6.8 \mathrm{~W}$ \\
Slope of energy consumption & 4 \\
Path loss model & $140.7+36.7 \mathrm{log}(d)$ \\
Noise power & $-111.45 \mathrm{dBm}$ \\
Shadowing model & Lognormal N $(0,8 \mathrm{~dB})$ \\
$\gamma_{\text {th }}$ & $256 \mathrm{Kbps}$ \\
\hline
\end{tabular}

Let us consider the other case that $u$ and $i$ are not associated with by the stable matching $\mu_{s}$, and $u$ prefers $i$ to the cell $j$ that it is currently associated with. Since $u$ likes $i$ more than $j, i$ must be acceptable to $u$. Moreover, $u$ must have attempted to be associated with $i$ before it tried $j$. Since $\mu_{s}$ does not associate $u$ with $i$, it means that $i$ rejected the association request from $u$ and accepted the association request from other UE $v$ who is acceptable and at least as preferable as $u$ to $i$. Thus, $\mu_{s}$ associates a cell $i$ with a UE acceptable to $i$. Since $\mu_{s}$ associates $u$ with $j, u$ and $j$ are mutually acceptable to each other. There-
TABLE 3: Proportion of cells whose energy efficiencies are less than $x$.

\begin{tabular}{lcccc}
\hline$\lambda_{\text {ue }}$ & $x$ & $0.5 \mathrm{Mbps} / \mathrm{W}$ & $1 \mathrm{Mbps} / \mathrm{W}$ & $4 \mathrm{Mbps} / \mathrm{W}$ \\
\hline \multirow{3}{*}{$200 / Y$} & Proposed & 0.53 & 0.69 & 0.96 \\
& SSBM & 0.53 & 0.72 & 0.98 \\
& DRBM & 0.53 & 0.72 & 0.98 \\
\multirow{2}{*}{$400 / Y$} & Proposed & 0.32 & 0.52 & 0.93 \\
& SSBM & 0.33 & 0.57 & 0.97 \\
& DRBM & 0.35 & 0.57 & 0.97 \\
$800 / Y$ & Proposed & 0.13 & 0.35 & 0.92 \\
& SSBM & 0.16 & 0.49 & 0.98 \\
& DRBM & 0.20 & 0.50 & 0.99 \\
\hline
\end{tabular}

fore, in this case, the UEs and the cells are associated with their mutually acceptable counterparts.

If a cell $i$ is not acceptable to a UE $u, u$ would stay unassociated rather than be associated with $i$. Thus, $u$ will not be associated with $i$ by the $\mu_{s}$. Likewise, if $u$ is not acceptable to $i$, $i$ will reject the association request if it receives one from $u$. Therefore, $\mu_{s}$ does not make an association between a UE and a cell if they are not mutually acceptable.

Since UEs and cells are associated with their mutually acceptable counterparts, they do not change the current association, which makes the small cell network stable.

\section{Simulation Results and Discussions}

In this section, we compare the performance of the proposed method with that of two alternative association methods under the same simulation environments. To make the 


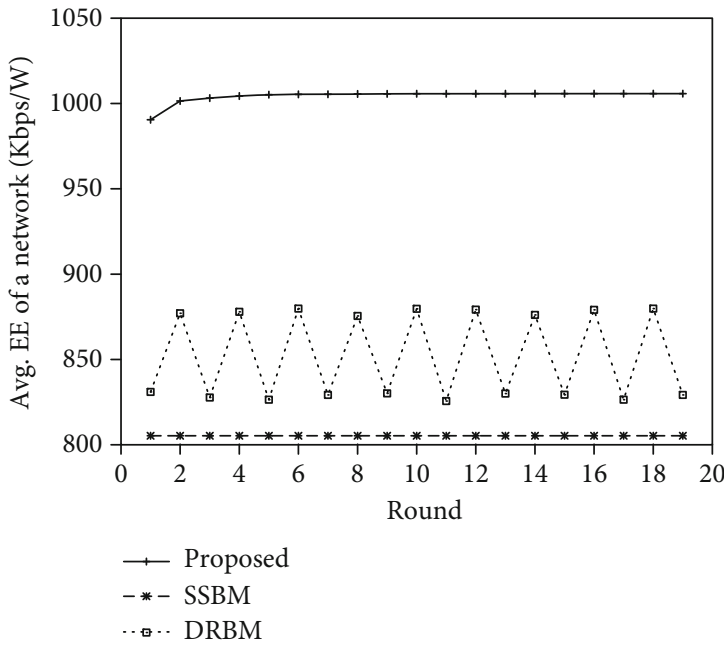

(a) $\lambda_{\text {ue }}=200 / Y$

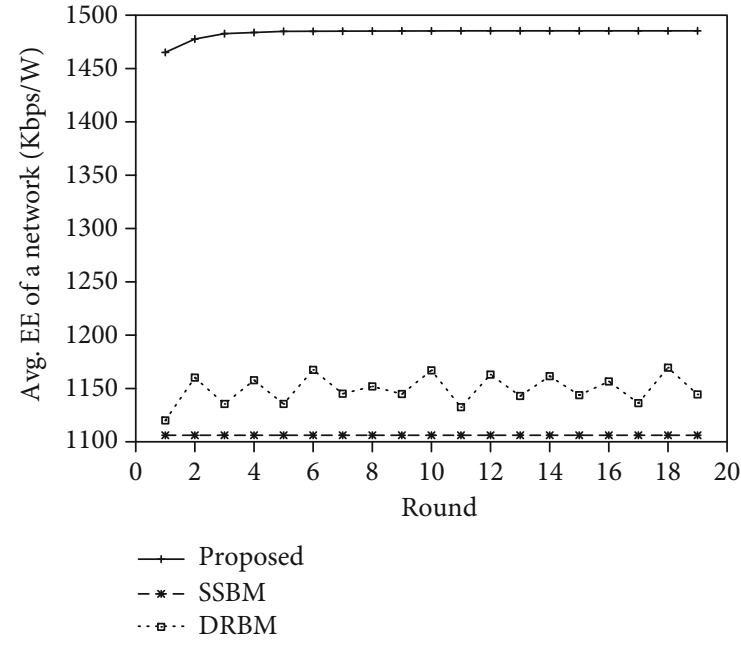

(b) $\lambda_{u e}=400 / Y$



(c) $\lambda_{u e}=800 / Y$

Figure 1: Comparison of the average network energy efficiency.

performance comparison, we make an event-driven simulator by using $\mathrm{C}$ language. The simulator is implemented in a cygwin on a windows 10 Pro for workstations. To run the simulator, we use a Dell precision 7920 workstation which has Intel Xeon(R) gold $511812 \mathrm{C} / 24 \mathrm{~T} \mathrm{CPU}$ at $2.3 \mathrm{GHz}$ and $16 \mathrm{~GB} 2,666 \mathrm{MHz}$ DDR4 RAM.

The first alternative method uses $\beta_{u, i}$. A UE $u$ asks for an association to a cell $i$ who gives $u$ the highest SINR, and $i$ accepts the request unconditionally. The method is widely used in many wireless networks. Henceforth, we name the first alternative as SSBM (Signal-Strength-Based Method). The second association method attempts to maximize the downlink data rate provided to each UE. In this method, a UE makes an association request to a cell that can give it the highest $\gamma_{u, i}$ and a cell accepts any association request unconditionally. In this method, a UE keeps changing its associated cell whenever it finds another cell that can give it a higher data rate than that it currently receives. Hereafter, we will call the second method as DRBM (Downlink-RateBased Method).
We deployed small cells in $100 \times 100 \mathrm{~m}^{2}$ region according to the homogeneous point Poisson process with mean $\lambda_{s c}$. We denote by $Y$ the simulation area. We also deployed UEs in the same region according to the homogeneous point Poisson process with mean $\lambda_{u e}$. The small cell network is configured according to $[16,17]$. We set the system bandwidth to $5 \mathrm{MHz}$ and $B_{P}=180 \mathrm{KHz}$. Thus, each small cell has $B_{M}=25$ PRBs. The transmission power of a cell is set to $21 \mathrm{dBm}$. The antenna gain of a cell is configured as $3.0 \mathrm{dBi}$ and that of a UE is set to $2.0 \mathrm{dBi}$. Each cell serves the UEs associated with it in a round-robin manner. We set the number of antennas in a cell $\left(n_{A}\right)$ to $1, P_{0}=6.8 \mathrm{~W}$, and $\Delta=4$. The path loss is configured as $140.7+36.7 \log (d)$, where $d$ is the distance between a cell and a UE. We also configure the noise power of $-111.45 \mathrm{dBm}$ and the log-normal shadowing with zero mean and variance of $8 \mathrm{~dB}$. When our method is used, we set $\gamma_{\text {th }}=256 \mathrm{Kbps}$. After setting $\lambda_{s c}=$ $200 / Y$, we observe how each method matches the set of UEs to the set of small cells by varying $\lambda_{u e}$. We summarize the simulation parameters in Table 2 . 


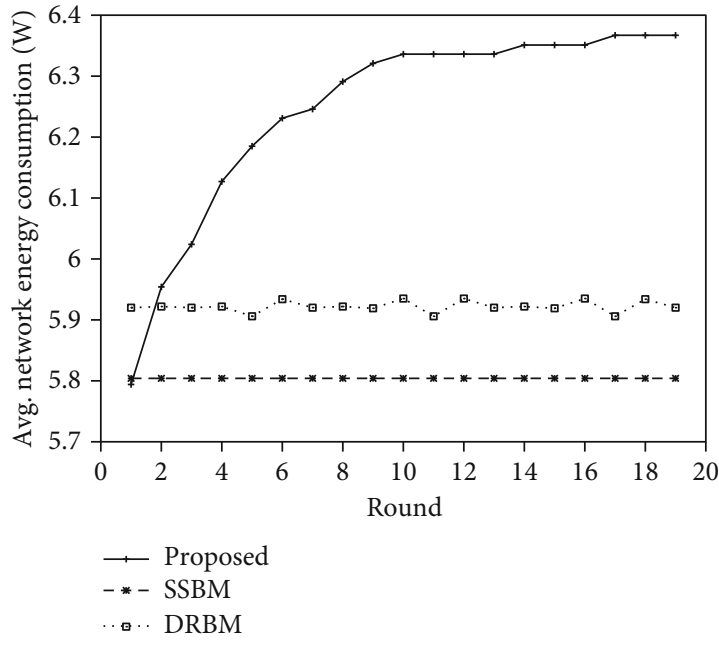

(a) $\lambda_{u e}=200 / Y$

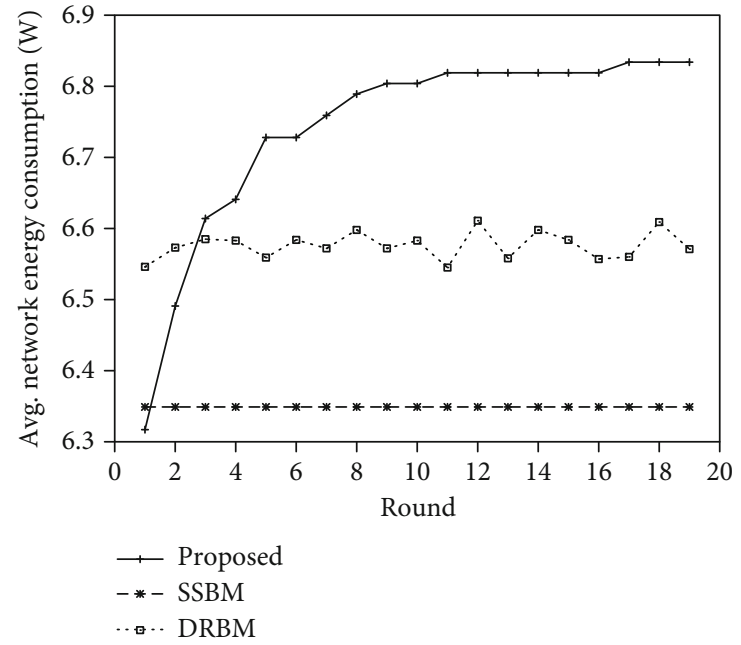

(b) $\lambda_{u e}=400 / Y$

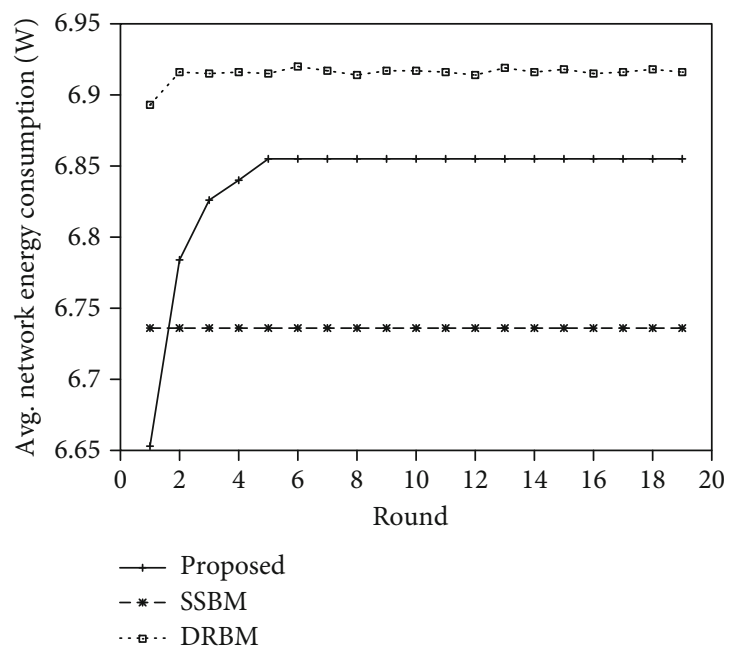

(c) $\lambda_{u e}=800 / Y$

FIGURE 2: Comparison of the average network energy consumption.

We measure the energy efficiency of each cell $\left(\Psi_{i}, \forall i \in C\right)$ and present the proportion of the cells whose energy efficiencies are less than $x$ in Table 3 . We can observe in the table that the energy efficiencies of the cells obtained by the proposed method are higher than those acquired when SSBM and DRBM are used. When $\lambda_{u e}=400 / Y, 52 \%$ of the small cells have less than $1 \mathrm{Mbps} / \mathrm{W}$ of $\mathrm{EE}$ when the proposed method is used. On the contrary, the proportion is increased to $57 \%$ when SSBM and DRBM are used. In the table, we can also observe that the energy efficiency gap between the proposed method and the other methods increases as the number of UEs increases. For example, we inspect the proportion of the cells whose energy efficiency is less than $1 \mathrm{Mbps} / \mathrm{W}$ (denoted by $\zeta$ ). When $\lambda_{u e}=200 / Y, \zeta=0.72$ when SSBM and DRBM are used while the proposed method reduces $\zeta$ to 0.69 . When $\lambda_{u e}$ increases to $800 / Y$, SSBM results in $\zeta=0.49$ and DRBM gives $\zeta=0.50$. On the contrary, the proposed method decreases $\zeta=0.35$, which means that $75 \%$ of the cells have $\Psi_{i} s$ higher than $1 \mathrm{Mbps} / \mathrm{W}$ (approximately $25 \%$ increase compared with the other methods).

In Figure 1, we compare the average network energy efficiency, $\bar{\Omega}=\Omega /|C|$. In this figure, the $x$-axis represents the number of round. We define the round as the time interval for all the UEs in $U$ which are not associated with any cell to make an association attempt once. In this figure, we can observe that the proposed method stabilizes the average network energy efficiency because it finds such pairs of UEs and cells that no UE in $U$ and no cell in $C$ want to change its associated counterpart. We can also observe that the proposed method results in higher network energy efficiency for all $\lambda_{u e}$, compared with SSBM and DRBM.

To scrutinize the results, we inspect the average energy consumption in the network $\left(\bar{E}=\sum_{i \in C} E_{i} /|C|\right)$ and the average downlink data rate provided by the network $\left.\left(\bar{\Gamma}=\sum_{i \in C} \Gamma_{i} /|C|\right)\right)$ in Figures 2 and 3, respectively. In Figure 2, we observe that $\bar{E}$ obtained after the proposed method is stabilized is larger than 


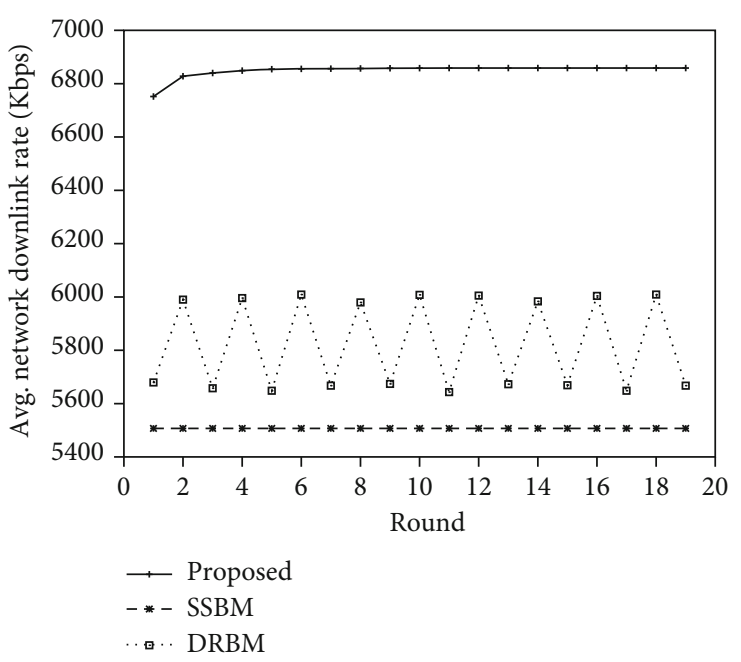

(a) $\lambda_{u e}=200 / Y$

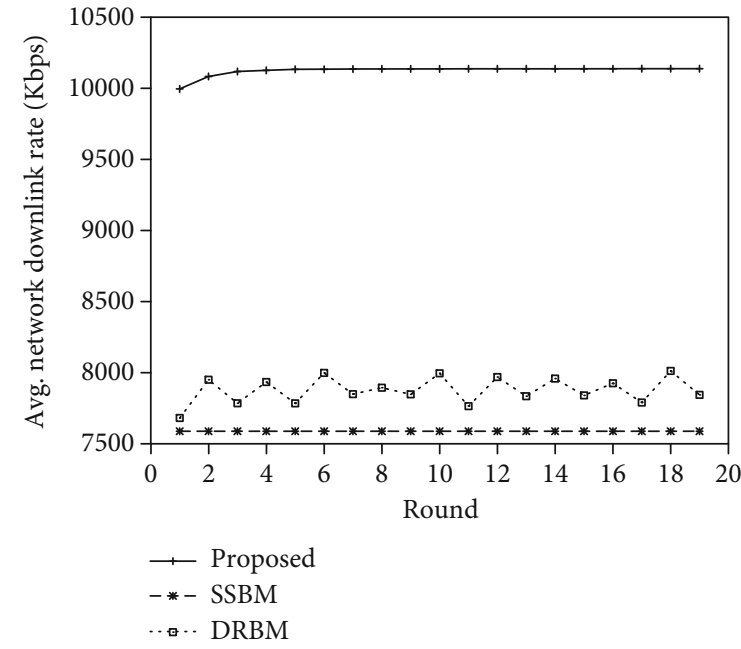

(b) $\lambda_{u e}=400 / Y$

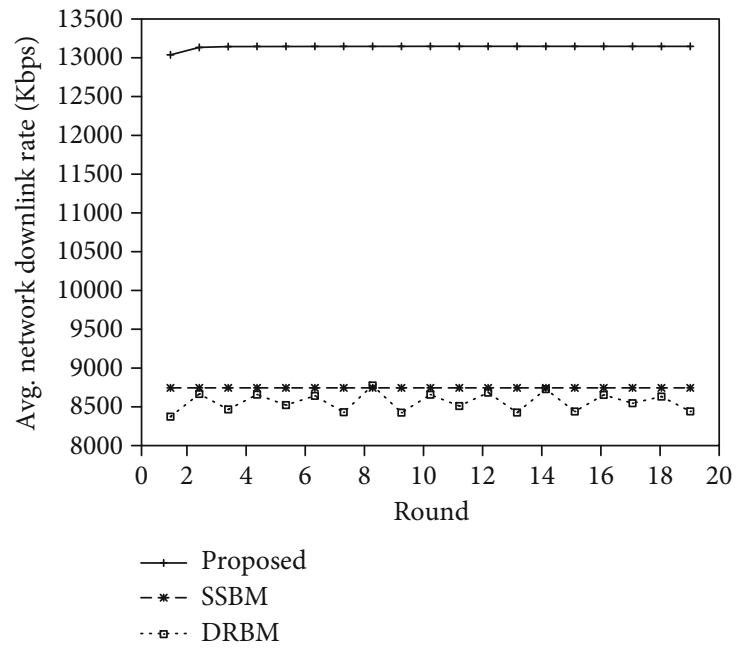

(c) $\lambda_{u e}=800 / Y$

Figure 3: Comparison of the average downlink data rate provided by the network.

those of SSBM and DRBM when $\lambda_{u e}=200 / Y$ and $\lambda_{u e}=400 / Y$. However, when $\lambda_{u e}$ increases to $800 / Y, \bar{E}$ of DRBM is larger than that of the proposed method. This is attributed to the fact that the proposed method distributes UEs to cells more evenly than the other methods. When SSBM and DRBM are used, UEs select their serving cells selfishly to increase their profits $\left(\beta_{u, i}\right.$ or $\left.\gamma_{u, i}\right)$ and cells accept the association requests from UEs unconditionally. Therefore, it is highly likely that some cells are crowded with UEs while the other cells are lightly loaded [18]. On the contrary, in the case of the proposed method, cells attempt to increase their profits (i.e., energy efficiencies) by determining whether to accept the association requests from UEs or not. Since UEs cannot change their serving cells unilaterally, the problem that UEs select the same cell at the similar time to overload the cell is reduced by the proposed method.

Therefore, when $\lambda_{u e} / \lambda_{s c}$ is small (Figures 2(a) and 2(b)), the number of cells that are not associated with any UE (i.e., $U_{i}=\varnothing$ ) is the smallest when the proposed method is used. Since a cell that does not serve a UE consumes the smallest energy and the UE density is small, $\bar{E}$ obtained by the proposed method are the highest in these environments. As the UE density increases, $\left|U_{i}\right|$ increases on average. Therefore, as we can observe in Figure 2(c), $\bar{E}$ produced by all the methods increase with $\lambda_{u e}$. However, the proposed method shows the smallest increase rate in $\bar{E}$, which makes $\bar{E}$ obtained by the proposed method smaller than that of DRBM when $\lambda_{u e}=800 / Y$.

When we inspect $\bar{\Gamma}$ in Figure 3, the proposed method outperforms the other methods. This is attributed to the fact that unlike the other methods, a cell using the proposed method resolves the contention among the UEs. The downlink data rate given to a UE depends not only on the SINR between a UE and a cell but also on the load of a cell serving the UE. Since SSBM does not consider cell loads, it is likely that some cells are highly congested while the other cells are lightly loaded, which results in the lowest average network downlink rate. When DRBM is used, a UE keeps changing its associated cell selfishly whenever it finds that the other 
TABLE 4: Proportion of UEs whose $\gamma_{u, i}$ are less than $x$.

\begin{tabular}{lcccc}
\hline$\lambda_{u e}$ & $x$ & $2 \mathrm{Mbps}$ & $5 \mathrm{Mbps}$ & $10 \mathrm{Mbps}$ \\
\hline \multirow{3}{*}{$200 / Y$} & Proposed & 0.32 & 0.58 & 0.81 \\
& SSBM & 0.29 & 0.68 & 0.87 \\
& DRBM & 0.27 & 0.67 & 0.87 \\
$400 / Y$ & Proposed & 0.32 & 0.59 & 0.81 \\
& SSBM & 0.46 & 0.80 & 0.92 \\
& DRBM & 0.44 & 0.80 & 0.92 \\
$800 / Y$ & Proposed & 0.29 & 0.61 & 0.83 \\
& SSBM & 0.67 & 0.90 & 0.97 \\
& DRBM & 0.65 & 0.91 & 0.98 \\
\hline
\end{tabular}

cell can give it higher $\gamma_{u, i}$ than that it currently receives. Therefore, we can observe in Figure 3 that $\bar{\Gamma}$ fluctuates over time. Unlike these methods where a cell accepts an association request unconditionally, a cell $i$ using the proposed method checks $\gamma_{\text {th }}$ provided to the UEs in $U_{i}$ and $\Psi_{i}$ when it decides whether to accept a new association request or not. Therefore, the proposed method can achieve higher $\bar{\Gamma}$ than those of SSBM and DRBM by resolving the contention among UEs. Since the gain obtained in $\bar{\Gamma}$ outweighs the loss occurred in $\bar{E}, \bar{\Omega}$ acquired by the proposed method becomes larger than those of the other methods.

We also inspect the proportion of UEs whose $\gamma_{u, i}$ are less than $x$. We denote such a proportion as $v(x)$. We measure $v(x)$ obtained by each method after the 15-th round and present them in Table 4 with different $x$. We observe that $v$ $(x)$ obtained by the proposed method is lower than those acquired by the other methods for all $x$ and $\lambda_{u e}$ except $\lambda_{u e}$ $=200 / Y$ and $x=2 \mathrm{Mbps}$. In this case, $v(2 \mathrm{Mbps})$ obtained by the proposed method is 0.32 which is higher than those obtained by the other methods. However, as $\lambda_{u e}$ and $x$ increase, the proposed method achieves smaller $v(2 \mathrm{Mbps})$ than the other methods. For example, when $\lambda_{u e}=800 / Y$ and $x=5 \mathrm{Mbps}$, only $61 \%$ of UEs receive less than $5 \mathrm{Mbps}$ from the network when the proposed method is used. However, when SSBM and DRBM are used, 90\% of UEs receive less than $5 \mathrm{Mbps}$. This is attributed to the fact that, as $\lambda_{u e}$ increases, more UEs compete for the network resources. Since UEs act selfishly to increase their $\gamma_{u, i}$ when SSBM and DRBM are used, they cannot resolve the contention. Therefore, they are associated with a cell that cannot give them higher $\gamma_{u, i}$ that they can get through a matching game. We also observe in the table that the difference between the $v(x)$ obtained by the proposed method and those acquired by SSBM and DRBM becomes larger as the number of UEs in the network increases.

\section{Conclusions and Future Works}

In this paper, we proposed an association algorithm that matches a set of UEs to a set of cells. Since the proposed algorithm considers $\gamma_{u, i}$, it can increase the proportion of UEs who receive higher downlink data rates from the network, compared with the SSBM and DRBM. In addition, the proposed method enhances the proportion of cells who have higher energy efficiency than those obtained by SSBM and DRBM because it also considers $\Psi_{i}$ when the association is made. As a future work, we are planning to expand the association method to satisfy the requirements imposed on the small cell networks to serve massive IoT devices.

\section{Data Availability}

All the data used in this manuscript are included in the manuscript.

\section{Conflicts of Interest}

The author declares that there is no conflict of interest regarding the publication of this paper.

\section{Acknowledgments}

This work was supported by Research Resettlement Fund for the new faculty of Kwangwoon University in 2019 and by the National Research Foundation of Korea (NRF) grant funded by the Korea Government (NRF-2018R1D1A1B07050893).

\section{References}

[1] Ericsson, Ericsson Mobility Report, Ericsson, 2019.

[2] M. G. Kibria, K. Nguyen, G. P. Villardi, K. Ishizu, and F. Kojima, "Next generation new radio small cell enhancement: architectural options, functionality and performance aspects," IEEE Wireless Communications, vol. 25, no. 4, pp. 120-128, 2018.

[3] C. D. Nwankwo, L. Zhang, A. Quddus, M. A. Imran, and R. Tafazolli, "A survey of self-interference management techniques for single frequency full duplex systems," IEEE Access, vol. 6, pp. 30242-30268, 2018.

[4] I. Afolabi, T. Taleb, K. Samdanis, A. Ksentini, and H. Flinck, "Network slicing and softwarization: a survey on principles, enabling technologies, and solutions," IEEE Communications Surveys and Tutorials, vol. 20, no. 3, pp. 2429-2453, 2018.

[5] F. Al-Turjman, E. Ever, and H. Zahmatkesh, "Small cells in the forthcoming 5G/IoT: traffic modelling and deployment overview," IEEE Communications Surveys and Tutorials, vol. 21, no. 1, pp. 28-65, 2019.

[6] S. K. Sharma and X. Wang, "Toward massive machine type communications in ultra-dense cellular IoT networks: current issues and machine learning-assisted solutions," IEEE Communications Surveys and Tutorials, vol. 22, no. 1, pp. 426471, 2020.

[7] M. Kamel, W. Hamouda, and A. Youssef, "Ultra-dense networks: a survey," IEEE Communications Surveys and Tutorials, vol. 18, no. 4, pp. 2522-2545, 2016.

[8] C. Liu, B. Natarajan, and H. Xia, "Small cell base station sleep strategies for energy efficiency," IEEE Transactions on Vehicular Technology, vol. 65, no. 3, pp. 1652-1661, 2016.

[9] R. Tao, W. Liu, X. Chu, and J. Zhang, "An energy saving small cell sleeping mechanism with cell range expansion in heterogeneous networks," IEEE Transactions on Wireless Communications, vol. 18, no. 5, pp. 2451-2463, 2019. 
[10] J. Zheng, Y. Wu, N. Zhang, H. Zhou, Y. Cai, and X. Shen, "Optimal power control in ultra-dense small cell networks: a game-theoretic approach," IEEE Transactions on Wireless Communications, vol. 16, no. 7, pp. 4139-4150, 2017.

[11] S. Mishra and C. S. R. Murthy, "Increasing energy efficiency via transmit power spreading in dense Femto cell networks," IEEE Systems Journal, vol. 12, no. 1, pp. 971-980, 2018.

[12] Y. Wang, S. Chen, H. Ji, and H. Zhang, "Load-aware dynamic biasing cell sssociation in small cell networks," 2014 IEEE International Conference on Communications (ICC), 2014, pp. 2684-2689, Sydney, NSW, Australia, 2014.

[13] A. J. Fehske, H. Klessig, J. Voigt, and G. P. Fettweis, "Concurrent load-aware adjustment of user association and antenna tilts in self-organizing radio networks," IEEE Transactions on Vehicular Technology, vol. 62, no. 5, pp. 1974-1988, 2013.

[14] G. Auer, V. Giannini, C. Desset et al., "How much energy is needed to run a wireless network?," IEEE Wireless Communications Magazine, vol. 18, no. 5, pp. 40-49, 2011.

[15] A. E. Roth and M. Sotomayor, "Chapter 16 Two-sided matching," Handbook of Game Theory with Economic Applications, vol. 1, 1992.

[16] 3GPP, Radio Frequency (RF) System Scenarios (Release 15), 3GPP TR 36.942 V15.0.0, The 3rd Generation Partnership Project (3GPP), 2018.

[17] 3GPP, Further Advancements for E-UTRA Physical Layer Aspects (Release 9), 3GPP TR 36.814 V9.2.0, The 3rd Generation Partnership Project (3GPP), 2018.

[18] Q. Ye, "User association for load balancing in heterogeneous cellular networks," IEEE Transactions on Wireless Communications, vol. 12, no. 6, pp. 2706-2716, 2013. 